Letter to the editor about "How effective is trauma simulation as an educational process for healthcare providers within the trauma networks? A systematic review"

\author{
Alexandra Lapierre, RN, MSc, PhD student ${ }^{1,3}$ \\ alexandra.lapierre@umontreal.ca \\ Jérôme Gauvin-Lepage, $\mathrm{RN}, \mathrm{PhD}^{1,2}$ \\ jerome.gauvin-lepage@umontreal.ca
}

Caroline Arbour, RN, PhD ${ }^{1,3}$

caroline.arbour@umontreal.ca

${ }^{1}$ Faculty of Nursing, Université de Montréal, 2375 Côte Ste-Catherine Road, Montreal (Quebec), Canada, H3T 1A8

${ }^{2}$ Centre de réadaptation Marie Enfant - CHU Sainte Justine Research center, 5200 Rue Bélanger, Montréal (Quebec), Canada, H1T 1C9

${ }^{3}$ Montreal Sacré-Coeur Hospital Research center, 5400 Boul. Gouin, Montreal (Quebec), Canada, H4J 1C5

This is the final version of the manuscript accepted for publication in the International Emergency Nursing Journal. The published version is accessible here:

https://doi.org/10.1016/j.ienj.2019.03.003 


\section{Letter to the Editor about "How effective is trauma simulation as an educational process for healthcare providers within the trauma networks? A systematic review".}

We have read with interest the review written by Barleycom and Lee published in the September issue of the International Emergency Nursing [1]. Systematic reviews are considered the 'gold-standard' form of evidence for assessing the impact of any teaching intervention. Aside from a focussed research question, a systematic review should comprise a comprehensive study selection strategy, data extraction plan, and proper assessment of the risk of bias. Although we agree with Barleycom and Lee on the importance to conduct a systematic review for the critical appraisal of simulation as an educational medium in trauma care, we would like to point out some methodological limitations that may affect the study conclusions.

\section{Study selection}

The value of any systematic reviews depends heavily on the quantity, quality, and heterogeneity of the included studies. In the review by Barleycom and Lee, we question the choice to exclude simulation studies published before 2010. The authors justify this decision on the fact that Major Trauma Networks were introduced in the UK from 2010. On the other hand, many others industrialized countries such as Canada and the United States have had established trauma Networks for several decades [2]. In fact, medical simulator that can mimic physiologic responses and provide real-time feedback have been widely available for practice and medical research since the '90 [3]. Although simulation equipment has greatly evolved in recent years, simulation-based learning as a general phenomenon remains the same [4]. For this reason, the exclusion of studies prior to 2010 may have brought an important selection bias in the review. Adding to the concern, two of the studies included in this systematic review were not specifically designed to assess the effects of a simulation intervention. Indeed, Briggs et al. (2015) [5] examined the relationship between non-technical skills and clinical performance in context of simulation but did not evaluated the impact of the simulation on any outcome measures. Likewise, Pucher et al. (2014) [6] assessed the non-technical skills and clinical performance of the teams in clinical practice itself but no actual simulation intervention has been studied.

\section{Data extraction and synthesis}

Extracting data from individual studies to be included in a systematic review is a relatively linear process. Still, organizing the data to give readers an overview of the important information in a meaningful manner is not an easy task. In the review by Barleycom and Lee, we believe extracted information should have been organized by types of simulation to get a better sense of their respective effects. In its present form, the review presents the simulation outcomes without making any distinction about the different types of simulation approach (like in situ, low or high fidelity, and team simulation). In reality, these simulation approaches generally have different learning objectives [7], an aspect that was not highlighted in the review. For instance, the use of an arm of a manikin dedicated to the installation of catheters (referring to low fidelity simulation) can be used for the acquisition of technical skills, but is less relevant for the acquisition of non-technical skills [7]. In the same way, some simulation interventions included in the review were performed individually while others were made as a team, even though the learning processes and learning objectives may be different. Furthermore, all the interventions included in this review have incorporated a didactical phase that is important to consider. As an example, Miller et al. (2012) [8] presented that $80 \%$ of the team members were reached by the didactic intervention, when only 
$25 \%$ were reached by the simulation intervention. With this information in mind, can we really consider that the simulation intervention is the cause of the results obtained? On a minor note, there is great variation in the outcomes that have been used in the reviewed studies to assess the effectiveness of trauma simulation. We noticed that, in some studies, team performance, task completion and non-technical skills were evaluated in a simulated environment (e.g. laboratory) while others directly in real care units. Since those results have been combined, it is creating confusion, as these outcomes are not equivalent. We believe that consideration of these elements in the presentation and discussion of extracted data would have permitted a better understanding of the different levels of impact of trauma simulation.

\section{Risk of bias}

About risk of bias assessment, the authors state that they used the Cochrane risk-of-bias tool [9]. However, a comprehensive text description or summary of the relevant trial characteristic on which judgments of risk of bias were based are lacking from Table 4, leading to a lack of transparency in how judgments were reached. Similarly, the specific domains of bias that were violated for each study were not specified. Another source of bias that is sometimes considered in systematic reviews is the source of funding [10]. This aspect is not included in the Cochrane riskof-bias tool. As simulation laboratories (in both clinical and educational settings) are often sponsored by external agencies, financial conflicts of interest can influence outcomes of trials in a way that is favourable to the sponsor. This aspect should have been taken into consideration.

In light of their work, Barleycom and Lee concluded that « from these, albeit, limited studies it is evident that trauma simulation is an effective educational tool, which can aid trauma learning, develop team's non-technical skills, increase task completion and indirectly have a positive impact on patient outcomes » (p.44). Considering the number of points with which we take issue in the review, we believe that it would be precipitated to statute about the effectiveness of simulation in trauma care. It is our belief that simulation in the trauma context is a very promising avenue for healthcare professional to develop their competences and performance. However, we think that this systematic review would need independent replication and bonification to really expose the effects of simulation as an educational process for healthcare providers within the trauma networks.

\section{Declaration of interest}

The authors report no declarations of interest. The authors alone are responsible for the content and writing of the paper.

Alexandra Lapierre, Jérôme Gauvin-Lepage,

Caroline Arbour

E-mail address: alexandra.lapierre@umontreal.ca

Faculty of Nursing, University of Montreal, 2375 Côte Ste-Catherine Road, Montreal (Quebec),

Canada, H3T 1 A 8 


\section{References}

[1] Barleycorn D, Lee GA. How effective is trauma simulation as an educational process for healthcare providers within the trauma networks? A systematic review. International emergency nursing. 2018;40:37-45.

[2] Hofman M, Pape HC. Trauma care systems. In: Oestern H-J, Trentz O, Uranues S, editors. General trauma care and related aspects. New Delhi, India: Springer-Verlag Berlin Heidelberg; 2014.

[3] Jones F. Simulation in Medical Education: Brief history and methodology. Principles and Practice of Clinical Research. 2015;1(2).

[4] Lateef F. Simulation-based learning: Just like the real thing. Journal of emergencies, trauma, and shock. 2010;3(4):348-52.

[5] Briggs A, Raja AS, Joyce MF, Yule SJ, Jiang W, Lipsitz SR, et al. The role of nontechnical skills in simulated trauma resuscitation. Journal of surgical education. 2015;72(4):732-9.

[6] Pucher PH, Aggarwal R, Batrick N, Jenkins M, Darzi A. Nontechnical skills performance and care processes in the management of the acute trauma patient. Surgery. 2014;155(5):902-9.

[7] Boet S, Savoldelli G, Granry JC. La simulation en santé : De la théorie à la pratique. Paris, France: Springer; 2013.

[8] Miller D, Crandall C, Washington III C, McLaughlin S. Improving Teamwork and Communication in Trauma Care Through In Situ Simulations. 2012;19(5):608-12.

[9] Higgins JPT, Green S. Cochrane Handbook for Systematic Reviews of Interventions Version 5.1.0: The Cochrane Collaboration; 2011. Available from: www.handbook.cochrane.org.

[10] Bekelman JE, Li Y, Gross CP. Scope and impact of financial conflicts of interest in biomedical research: a systematic review. Jama. 2003;289(4):454-65. 